\title{
Learning Visual Ideals
}

\author{
M. Burge and W. Burger \\ Johannes Kepler University \\ Department of Systems Science \\ Computer Vision Laboratory \\ A-4040 Linz, Austria \\ burge@cast.uni-linz.ac.at
}

\begin{abstract}
We address the problem of describing, recognizing, and learning generic, free-form objects in real-world scenes. We introduce a hybrid appearance-based approach, IDEAL, where objects are encoded as a loose collections of parts and the relations between them. The key features of this new approach are the structural part decomposition combining multi-scale wavelet segmentation and hierarchical blobs, and learning to recognize generic object categories, exhibiting large intra-class variability, from real examples with automatic model acquisition.
\end{abstract}

Then, my dear friend, must not the law-giver also know how to embody in the sounds and syllables that name which is fitted by nature for each object? Must he not make and give all his names with his eye fixed upon the absolute or ideal name, if he is to be an authoritative giver of names? And if different lawgivers do not embody it in the same syllables, we must not forget this ideal name on that account; for different smiths do not embody the form in the same iron, though making the same instrument for the same purpose, but so long as they reproduce the same ideal, though it be in different iron, still the instrument is as it should be, whether it be made here or in foreign lands, is it not? - Socrates in Plato's Cratylus $§ 389 \mathrm{e}$

As Socrates explained to Hermogenes, we carry a conceptual ideal of many objects in our thoughts. Indeed the concept of ideals are embedded in our very languages, witness the almost universal usage of definite and indefinite articles. When we say "a chair", we envision in a sense the ideal form of a chair. One does not recall an actual chair, but instead a functional description of what a chair does combined with a relational-structural description of the parts which make up a chair. In our system, IDEAL[2,3], we attempt to learn from example objects the structural parts and the relations between them which when recognized in a previously unseen object, enable the system to probabilistically classify it amongst its known classes.

\section{3-D Object Recognition}

Recognizing three-dimensional objects under different viewing and lighting conditions is a traditional problem in computer vision. The difficulty of the problem 
depends upon both learning factors such as the types of objects, number of classes, and the inter- and intra-class variability, and imaging problems like the segmentation of multiple object scenes, background complexity and the amount of occlusion. Given the complexity of the problem many different solutions have been proposed, in table1 a a comparison of 24 different approaches is given. Nearly half of the approaches reviewed dealt with only a single object, and and those that dealt with many often did only under severe limitations on affine transformations and occlusions of the objects. Nearly all approaches reviewed featured rotational and translational invariance, but other affine transforms, i.e. scaling were handled in only a few. The recognition of occluded objects was treated by almost every approach, though in many cases the degree of occlusion acceptable was not apparent. ${ }^{1}$

\section{The IDEAL System}

We focus on learning to recognize single object scenes of classes (e.g., chair, table, bench, etc.) which often exhibit high intra-class variability. Our approach is appearance-based and structural, i.e., typical views of an objects are described in terms of the configurations of their parts The structural representation and recognition approach emphasizes the shape, spatial arrangement, and topology of a pattern providing tolerance against: variations in lighting conditions, object deformation, articulation, and occluded, missing, and extraneous parts.

Structural recognition methods are based on both the availability of primitive structural elements like: straight line segments, corners, blobs, arcs, and other parametric strokes, and on the assumption that these elements can be extracted from the image data with sufficient reliability. Regardless of what the primitives are, the performance of the recognition process depends critically on how reliably they can be extracted, which is difficult even under ideal viewing conditions. When images are noisy and cluttered, the extraction of suitable primitives using only local information may not be possible. In addition, practically all structural feature extraction schemes work in a rather myopic fashion, trying to assemble larger meaningful structures from scattered pieces using weak local evidence and therefor often missing the dominant global structures in the image description (segmentation). In practice, most current structure extraction schemes are fragile and no single method exists that can reliably deliver a good part decomposition, unless the scene domain is highly restricted. For these reasons many recognition approaches assume a noiseless, pre-segmented image with all parts of the object visible in every view, i.e. no self-occlusion, and models of all objects given $a$ priori.

Most structural recognition methods are based on a single type of primitive, commonly straight line segments. Some approaches combine different primitive types of the same class of primitives, e.g., a contour class representation might

${ }^{1}$ This summary is based upon approaches published from 1973-1992, a summary for 1993 to the present is being prepared, bibliographic references are limited to author and year due to space considerations. 


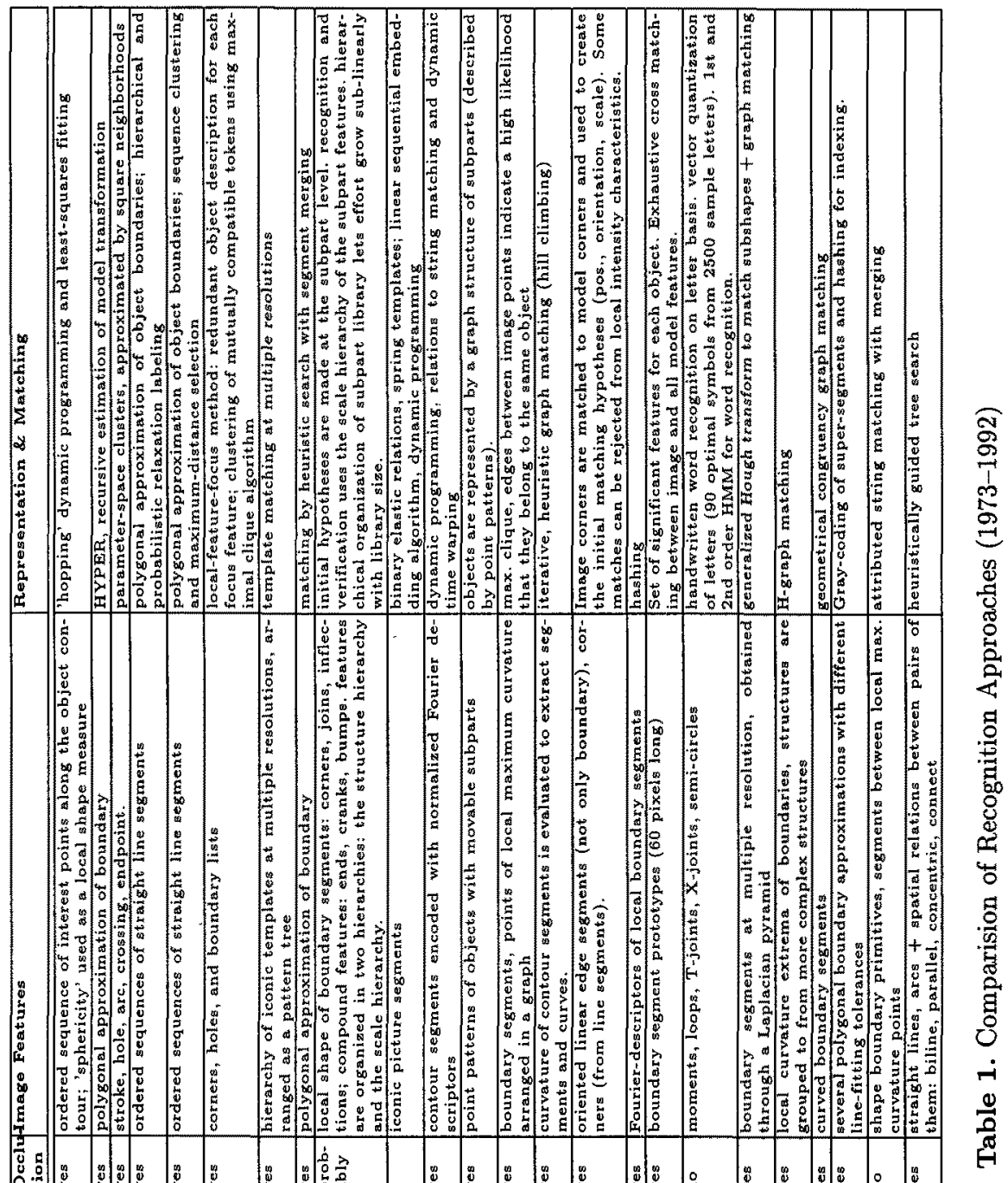

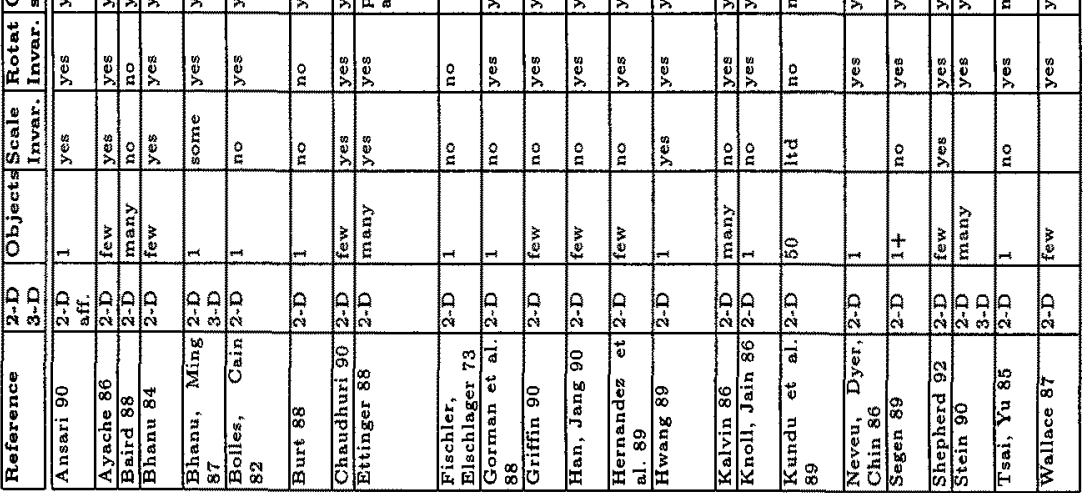


use both straight line segments in combination with circular arcs for primitives. The combination of different primitive classes, e.g., contour and area we refer to as polymorphic, previous work[3] gives an example of IDEAL using polymorphic primitives and this paper presents IDEAL using an area class representation.

We attempt to overcome the problem of fragile segmentation in three ways. First, instead of relying upon a single type of primitive, we combine two area based of primitives into a single representation and recognition scheme, introducing additional redundancy. Secondly we do not require structural primitives to be precisely delineated but only their approximate spatial position and shape properties are needed and parts may be overlapping and ambiguous. Finally structural primitives do not need to correspond to parts such as would be meaningful to a human observer, it is only necessary that they can be recovered reliably and repeatably from an image. The two area based features we use are Lindeberg's[5] blob features and a class of Gabor quadrature filters we developed.
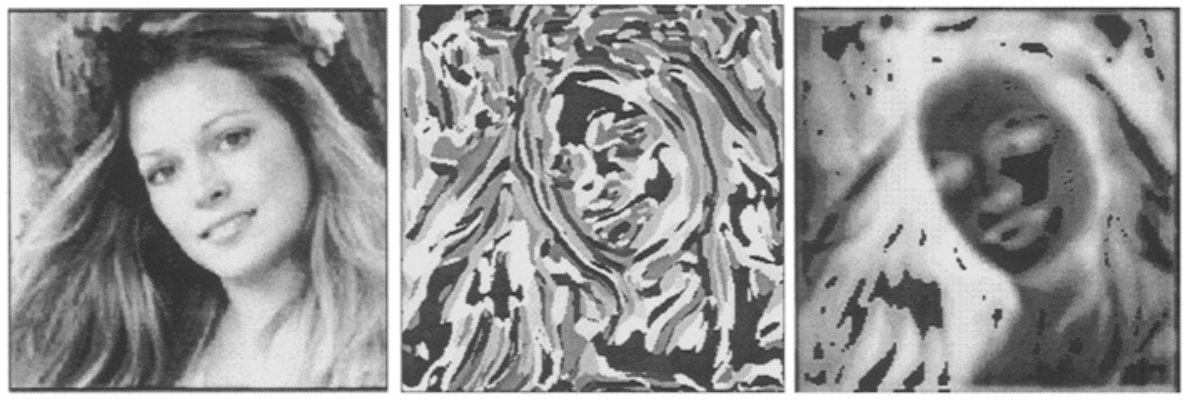

Fig. 1. Initial structural description Original, Gabor Probe, Hiearchical Blobs

A set of Gabor quadrature filters with $N_{\omega} \log$-spaced center frequencies $\omega_{k}$ and $N_{\phi}$ regularly spaced orientations $\phi_{l}$ [4]. For each pixel location $\mathbf{x}_{i}$, we compute the values $J_{k, l}^{+}[i]=\left(I * G_{\omega_{k}, \phi_{l}}^{+}\right)\left[\mathbf{x}_{i}\right]$ and $J_{k, l}^{-}[i]=\left(I * G_{\omega_{k}, \phi_{l}}^{-}\right)\left[\mathbf{x}_{i}\right]$ where $I$ denotes the original image, $\left(G_{\omega_{k}, \phi_{i}}^{+}, G_{\omega_{k}, \phi_{l}}^{-}\right)$is a Gabor quadrature filter pair with center frequency $\omega_{k}$ and modulation orientation $\phi_{l}$, * is the convolution operator, and $G_{\omega_{k}, \phi_{l}}^{+}$and $G_{\omega_{k}, \phi_{l}}^{-}$are respectively the cosine and the sine $\mathrm{Ga}$ bor filter kernels. The spacing in frequency is $\Delta_{\omega}$, such that $\omega_{k}=\omega_{0} \cdot \Delta_{\omega}^{k}$ for $1 \leq k \leq N_{\omega}$, and the spacing in orientation is $\Delta_{\phi}=\frac{\pi}{N_{\phi}}$, such that $\phi_{l}=\phi_{0}+l \cdot \Delta_{\phi}$ for $1 \leq l \leq N_{\phi}$, for given $\omega_{0}, N_{\omega}$, $\phi_{0}$, and $N_{\phi}$. We use values of $\omega_{0}=0.05 \pi$, $N_{\phi}=\overline{4}, \Delta_{\omega}=\sqrt{2}$, and $N_{\omega}=4$, to construct a 32-element Gabor probe, G[i], at each image location $\mathbf{x}_{i}$.

The output of this filter bank is a 32-element vector $D_{\omega, \phi}(\mathbf{x})$ (called a Gabor Probe) that describes the structure around the image location $\mathrm{x}$ at multiple scales. Subsequently, the Gabor probes are classified into $N$ (we use $N=64$ ) structural event types, using vector quantization with a codebook derived from a representative set of training images. Contiguous areas in the resulting label 
image $L(\mathbf{x}) \in[0, N-1]$ form a set $\mathcal{R}$ of regions with approximately homogeneous image structure (Figure 1). Each region $R_{i} \in \mathcal{R}$ is characterized by its shape and codebook index which describes the corresponding image structure in terms of the Gabor filter response and provides a set of unary region features for recognition.

\subsection{Part Compatibility Graph}

The problem of label compatibility arises when an object has the same parts and features as a different object. The addition of relations between parts, that is binary features, adds structural context. In recognition without labeled parts one must exhaustively attempt all matchings between unary and binary relations. If the relations are encoded as in a part adjacency graph (PAG) then it becomes a problem of subgraph isomorphism between unlabeled graphs. It is possible to avoid this prohibitively expensive matching problem by encoding the binary relations between the parts during learning in such a way that only label compatible part paths can be generated during both the learning and recognition stages.

Generating only label compatible part paths can be done by calculating the binary features only between neighboring parts of the same object while building the tree. The tree constructed in this way contains only paths from the root, where all parts are represented in some cluster, to the final classification leaves which correspond to the paths in the object from a part to its neighbor. The use of the neighboring relation as a constraint during both the training and recognition stages implicitly solves the label compatibility problem by assuring that any matched sequence must have arisen from a label compatible sequence. This solution is not sufficient since representative and therefor important sequences for recognition occur among non-neighboring parts scattered across the image. It is necessary to have a relation which still provides the constraints for solving the label compatibility problem and allows the non-neighboring representative parts to be combined into the same part paths.

In a PAG graph $G$ an edge $e_{i, j}$ is constructed between the parts $p_{i}$ and $p_{j}$ if the parts are neighbors. Neighborhoods are defined through either physical adjacency or, more generally, spatial proximity, e.g., parts within 10 pixels of each other. Other neighborhood definitions, for example Area Voronoi neighborhoods, are possible but all suffer from the drawback that simply being a neighbor of another part is not necessarily a significant relation for recognition. In a part compatibility graph (PCG) however, a graph edge $e_{i, j}$ denotes a high similarity measure between parts $p_{i}$ and $p_{j}$, that is, the similarity measure replaces distance as used in the PAG. The similarity function used is formulated in terms of the moments of the regions in the initial structural part decomposition and results in edge set growth of $P G C(|\mathcal{E}|) \approx 1.3 P A G(|\mathcal{E}|)$, for details of constructing the $\mathrm{PGC}$ for a given feature set see[3].

Increasingly techniques from machine learning are being incorporated into object recognition methods. Using the ability of these techniques to generalize from the training data previously unseen views can be recognized with increased accuracy. These include methods using the relative frequency of a class in a 
region as an evidence-based approach to generalization while others use neural networks, Eigenvalue decompositions, and decision trees which are the basis for learning in IDEAL.

\subsection{Automatic Model Acquisition and Recognition}

We use Bischof and Caelli's[1] decision-tree based Conditional Rule Generation (CRG) during training and classification it exhaustively generates neighboring part paths so that if during classification a part is missing, a path without that part might still be found in the classification tree and the object may still be correctly classified. Automatic model acquisition encodes the models into the rules of the decision tree as the basis of our learning and recognition method. several directions.

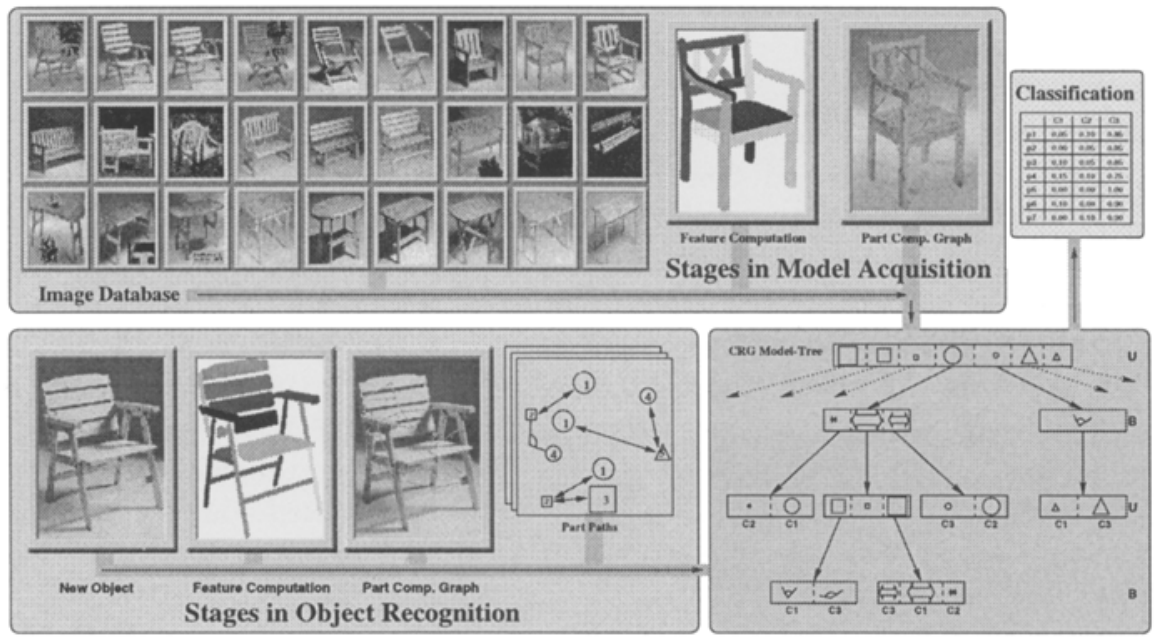

Fig. 2. Flowchart of the Learning and Recognition process: (a) Learning path: computation of the features from all images in the database; building of a part compatibility graph (PCG); construction of a classification tree for part paths. (b) Recognition process: presentation of a new image; feature computation; $P C G$; extraction of part paths; classification of each part path using the classification tree; combining evidence.

An attributed PCG graph, $G(\mathcal{P}, \mathcal{E}, \mathcal{U}, \mathcal{B})$, is constructed from the initial structural segmentation in which each segmented regions is considered a "part" $\mathcal{P}$ in $G$. These parts are connected by a set of edges $\mathcal{E}$ which represent relations between the parts. To avoid the polynomial number of edges in a fully connected graph, only edges between selected, in this case based upon part similarity, parts are included. Each part $p_{i} \in \mathcal{P}$ is attributed by a unary feature vector $u_{i} \in \mathcal{U}$ with a predetermined number of features, each edge $e_{i, j} \in \mathcal{E}$ is attributed by a binary feature vector $b_{i, j} \in \mathcal{B}$ whose features are computed from the parts $p_{i}$ 
and $p_{j}(i \neq j)$. The feature vectors $u_{i}$ and $b_{i, j}$ form the unary and binary feature spaces $\mathcal{U}$ and $\mathcal{B}$ respectively.

In the learning phase the problem is learning how to classify a part $p_{i}$ into the correct class $C\left(p_{i}\right)$. The training examples are presented separately and sequentially in a supervised fashion. The CRG method works by first classifying all unary features from all parts, of all views and all object classes into a unary feature space $U$. This feature space is then clustered into a number of clusters $U_{n}$, some of which may already be unique with respect to class membership, i.e. all parts in a cluster belong to the same class, $\exists_{c} \forall_{p \in U_{n}} C(p)=c$, while others will not. Binary features are then calculated between the parts of the non-unique clusters and its neighbors in the graph. The binary feature spaces resulting from the unresolved clusters are again clustered, each forming feature spaces of type $U_{n} B_{m}$. For the non-unique clusters the unary feature spaces $U_{n} B_{m} U_{o}$ of the parts of the previous binary relations are determined and clustered. This continues until all clusters are unique or a predetermined maximum depth has been reached.

Once the graph model of the object to be recognized has been constructed it must be "matched" against those in the database to determine the class in which it should be classified. One method of matching graph based models would be to determine the relational distance metric between the models. Even with new algorithms developed for parallel computers, unlabeled attributed graph matching is a computationally expensive process, with the general problem being NP complete. Instead of attempting to match the object graphs against the model graphs, i.e. finding subgraph isomorphisms, short part paths through the graph are matched. An evidence accumulation technique is used where a large number (dependent upon the object but typically in the range of $50-100$ ) of small (typically 3 to 5 nodes in length) paths are extracted starting from each node in the object's graph representation and then matched against the generic model.

In recognition the goal is to correctly classify using the CRG tree, the previously unseen objects with possibly occluded and missing parts. First a PAG graph similar to that used in the learning phase is constructed. In this graph all non-cyclic paths up to a maximum length of the depth of the decision tree are generated and then classified using the decision tree. For each path an evidence vector with the probability that this path belongs to a particular class is computed. During learning this evidence vector is computed using the relative class frequencies for this branch of the decision tree. The evidence vectors of all paths starting at some part $p_{i}$ determine its classification.

\section{Conclusion}

A structural appearance-based approach, IDEAL, for describing, recognizing, and learning generic, free-form objects in real-world scenes was introduced. An initial structural part decomposition based combining multi-scale wavelet segmentation and hierarchical blobs was presented. A method based on CRG for 

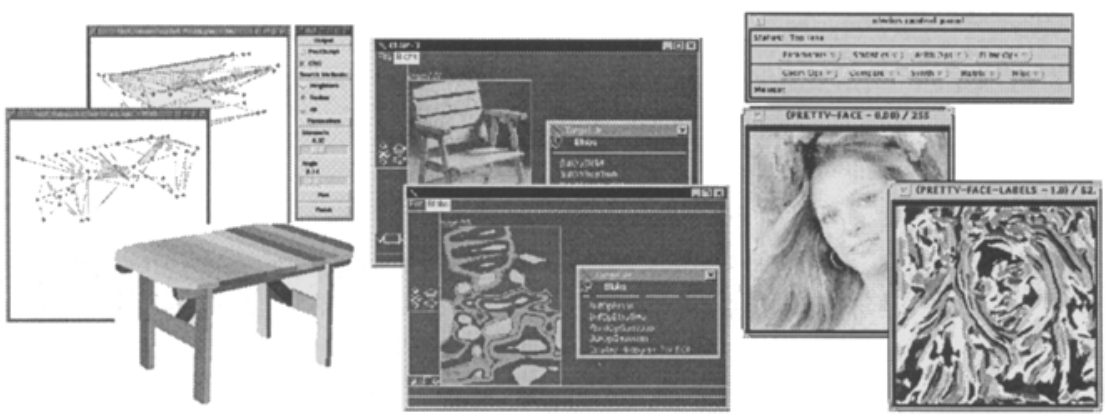

Fig. 3. IDEAL is implemented as a collection of interdependent UNIX processes, including: (a) Part path selection under TK-TCL in C+t, (b) Blob feature detector under Target Jr in $\mathrm{C}++$, (c) Local structure segmentation under Obvious in Cornmon Lisp.

recognizing generic object categories, exhibiting large intra-class variability, from real examples with automatic model acquisition was given. Our current research directions for IDEAL are primarily in the areas of machine learning where we are examining issues of class generalization and incremental learning.

IDEAL has been tested[3] on a images exhibiting real-world imaging problems such as shadowing and self occlusion, of generic classes e.g. chairs, benches, and tables, purposely selected to provide for a high degree of structural similarity between classes. The performance with occluded parts is a combination of missing and additional parts, as an occluded part generally causes a missing part and a number of additional parts to occur in the image. The system performs well even when their are considerable missing parts, this is due to part paths extracted by the proposed PCG method extend over larger regions of the object and are therefore more likely to contain non-neighboring significant parts, though performance drops when additional parts occurs. A more detailed analysis of these experiments can be found in[2].

\section{References}

1. W. Bischof and T. Caelli. Learning structural descriptions: a new technique for conditional clustering and rule generation. $P R$, pages 689-697, 1994.

2. M. Burge, W. Burger, and W. Mayr. Learning to recognize generic visual categories using a hybrid structural approach. In ICIP-96, v 2, pp 321-324, Switzerland, 1996.

3. M, Burge, W. Burger, and W. Mayr. Recognition and learning with polymorphic structural components. In 13th ICPR, v 1, pp 19-23, Vienna, Austria, 1996.

4. W. Burger and B. Bhanu. Signal-to-symbol conversion for structural object recognition using hidden Markov models. In Proc. ARPA Image Understanding Workshop, pages 1287-1291, Monterey, CA, 1994.

5. T. Lindeberg. Detecting salient blob-like image structures and their scales with a scale-space primal sketch. $I J C V, 11(3): 283-318,1993$. 